\title{
Agent-Based Modelling of a Commodity Market Dynamics
}

\author{
R. Gębarowski ${ }^{a, *}$, S. Drożdż $\dot{z}^{b}$, A.Z. Górski ${ }^{b}$ And P. OśWiȩcimkA ${ }^{b}$ \\ ${ }^{a}$ Institute of Physics, Cracow University of Technology, Podchorążych 1, 30-084 Kraków, Poland \\ ${ }^{b}$ Complex Systems Theory Department, The Henryk Niewodniczański Institute of Nuclear Physics, Polish Academy \\ of Sciences, E. Radzikowskiego 152, 31-342 Kraków, Poland
}

\begin{abstract}
A modification of Yasutomi's agent-based model of the commodity market is investigated. It is argued that introduced modification of the microscopic exchange rules allows for emergence of commodity exchange rates in the model. Moreover, the model scaling due to finite size effects is considered and some practical implications of such scaling are discussed.
\end{abstract}

DOI: 10.12693/APhysPolA.129.1032

PACS/topics: 89.65.Gh, 89.75.Fb, 05.45.Tp

\section{Introduction}

Agent-based computational models of economy offer an interesting possibilty for studying an application of physical concepts to financial and commodity markets [1]. Some of fruitful ideas taken from physics include scaling and criticality in a stochastic multi-agent models [2], thermodynamics and statistical physics of the markets $[3,4]$ and role of the Ising model of the phase transition for understanding social and financial systems [1].

Our aim is to study a variation of an agent-based computational model introduced by Yasutomi [5] twenty years ago. The model has been investigated later in follow-up research by Yasutomi himself [6] and revisited by Górski et al. (2010) [7]. Arguably Yasutomi's agent-based model of a commodity market could be perceived as a toy model rather than a fully fledged agentbased computational economics approach. Nonetheless, it proved to be quite useful for exposing some universal features of the complex systems in general, and economics markets in particular. Application of Yasutomi's approach to modelling the commodity market allowed to reproduce some real market behavior with statistical signatures of the money emergence phenomena $[8]$ as well as provided some evidence of the competition of commodities for a dominant status of the so-called commoditybased money [9].

In this work we will investigate a modification of the exchange protocol, allowing for a more flexible commodity exchange than just one-to-one barter swap. It is expected that the toy model for commodity market would become more realistic and would allow for emergence of varying in time exchange rates among commodities. In a realistic market the number of traders $N$ is much greater than the number of different types of commodities $M$. We also consider practical implications of having freedom to study numerically general cases $N \geq M$. This

*corresponding author; e-mail: rgebarowski@pk.edu.pl would allow for a fresh look at the problem of numerical convergence of results and finite size scalings, where dominant commodity becomes well pronounced.

In the following section, we describe the model used in our present research, with emphasis on introduced modifications. Then we present results of the numerical simulations with the focus on variation with time of the commodity strength and signatures heralding existence of a single dominant commodity on the market. We will also look at a possibility of finding some scalings due to the finite size of the studied model. Finally we present a summary and draw some conclusions.

\section{The agent-based computational model}

The model adopted in the present research is essentially a modification of an agent-based model introduced by Yasutomi (1995). We consider a new protocol of exchange commodities. Instead of a generic one-to-one exchange, a simple idea of the value-for-value exchange between trading agents is introduced. This is an attempt to include the fact that swapped commodities may have different unit values as the model commodity market evolves in time. This could in principle allow for our economics toy model to be more flexible with respect to emergence of natural commodity exchange rates in time, and therefore more suitable for the model dynamics investigation. Another important point emphasised by the present model is allowing for taking independently parameters describing the size of the model. Instead of having, as in previous works (see e.g. [9]), the same number of agents and commodity types, we allow now for considering these sizes independently. Indeed, in a real situation on the market we have many more traders than commodity types.

In the following subsection our present model is described in more detail. In the description we will emphasise introduced modifications.

\subsection{The description of the present model}

In the present agent-based model of the commodity market, there is an ensemble of $N$ trading agents and $M$ different types of commodities, which could be supplied 
and consumed by agents $(N \geq M)$. There are some microscopic rules for trading in this toy economic model, which allow to determine the dynamics of the model by means of the system observables, obtained by taking averages over statistical ensembles.

A generic single transaction on our toy commodity market consists of a few steps, including: a random choice of an agent, matching a co-trader for the chosen agent, their interaction by means of exchanging certain amount of commodities according to the preferences, averaged market opinion, as well as currently deemed value of swapped goods. Finally, there is a stage of the consumption and the production of the commodities. A round of $N$ such transactions (which is equal to the number of agents) is called a turn and sets a unit of time $t$.

Trading agents $k=1,2, \ldots, N$, are equipped with varying in time $t$ an integer number of $j$-th commodity $(j=1,2, \ldots, M)$ denoted by $P_{t}(j, k)$, randomly changing preferences $W_{t}(j, k)$ as to the most wanted commodity (in our model there is only one wanted commodity by an agent, who is not the supplier of that commodity) and evolving views (the market opinions) $V_{t}(j, k)$ on the value of any particular commodity type. The matched pair of the agent and its co-trader increase their own views on any commodity in case their demands were not satisfied in the previous transaction. Hence values $V_{t}(j, k)$ contain some sort of memory on the past transactions. Subsequently they average their mutual views on each particular commodity. Varying in time values $V_{t}(j, k)$ are normalized with respect to the total number of commodity types (denoted by $M$ ) in such a way that $1 \leq V_{t}(j, k) \leq M$. The number of commodity types $M$ is less than or equal to the number of agents. If $N>M$, more than one agent can supply or consume the same commodity.

The commodity $j$ enters the wish-list of the agent $k$ also when $V_{t}(j, k) \geq T$. That is, once a view on a particular commodity becomes equal to or greater than a certain macroscopic model parameter $T, V_{t}(j, k) \geq T$, the commodity $j$ becomes also wanted by a trading agent $k$. The global macroscopic activation of interest parameter is called the threshold parameter $T$. This threshold parameter serves therefore as an external, global market view on the degree of attractiveness of such commodity. Recall that each trader is also driven by its internal (individual and independent) need for a single randomly chosen commodity given by $W_{t}(j, k)$.

However, unlike previously implemented (see e.g. $[5,8,9])$, when both traders have an unequal number of commodities of different types on their wishlists, they consider the total value of the commodities to be exchanged. They exchange commodities on their wish-lists, starting from items of the highest value. They keep exchanging integer number of commodities until they swap approximately the same value from their demand-lists. The value of the commodity $j$ deemed by the $k$-th agent is proportional to the value of the view on that commodity $V_{t}(j, k)$ at a given turn $t$ shared by the $k$-th agent. The same commodity value evaluation made by $l$-th agent is based on its own view $V_{t}(j, l)$.

At the end of each transaction, the desired commodities are fully consumed (expended) by a trading pair of agents. If there is no self-supplied (produced) commodity in the portfolio of any of these two agents, then a unit of such commodity is produced. The statistical ensemble is created out of a certain number of random initial conditions for initially preferred commodity. Thus a number of "trajectories" for the system is obtained through dynamics arising from different $W_{0}(j, k)$ values in trading time measured in turns. Here it is also worth pointing out that for any given randomly chosen initial condition, there is also an additional stochastic component due to a random choice of pairs of trading agents as described above.

\subsection{The commodity strength}

Let us define the commodity strength $V_{\mathrm{CS}}^{(j)}(t)$ in a straightforward way [9], as normalized view on commodity $j$, averaged over the agent's ensemble

$$
V_{\mathrm{CS}}^{(j)}(t):=\frac{1}{N} \sum_{k} V_{t}(j, k) .
$$

If above defined commodity strength is maximized for a certain $j=j_{\text {max }}$ :

$$
V_{\max }(t):=\max _{j}\left\{V_{\mathrm{CS}}^{(j)}(t)\right\},
$$

than we can say that $j=j_{\max }$ commodity reached the status of the dominant commodity.

Therefore such $V_{\max }$ defines the dominant commodity strength, which could be regarded as a commodity based money as discussed in [7, 8].

\section{The results}

In the following, we will consider numerical simulations of the investigated model.

\subsection{The dominant commodity strength}

In order to study the dominant commodity strength (cf. Eq. (2)) originating in the model, we have made a statistical averaging over initial conditions ("trajectories") after certain time $t=R$, defining the range of time evolution observations.

In Fig. 1 the scaled strength of the money status versus threshold $T$ is shown for two cases, with numbers $N=100$ and $N=200$ of agents (here $M=N$ ) taken for the simulations of our model market. The money strength is averaged for $n r=100$ realizations after $R=1000$ turns of the evolution time in each case. The plot shows the influence of the exchange protocol modification. New data are denoted by filled points, whereas the data coming from the previously studied model [9] are denoted by open points. The dominant commodity strength $V_{\max }$ is rescaled here by the model size $N$ to make the comparison easier. Error bars show the dispersion of the numerically obtained data and correspond to a single standard deviation. 


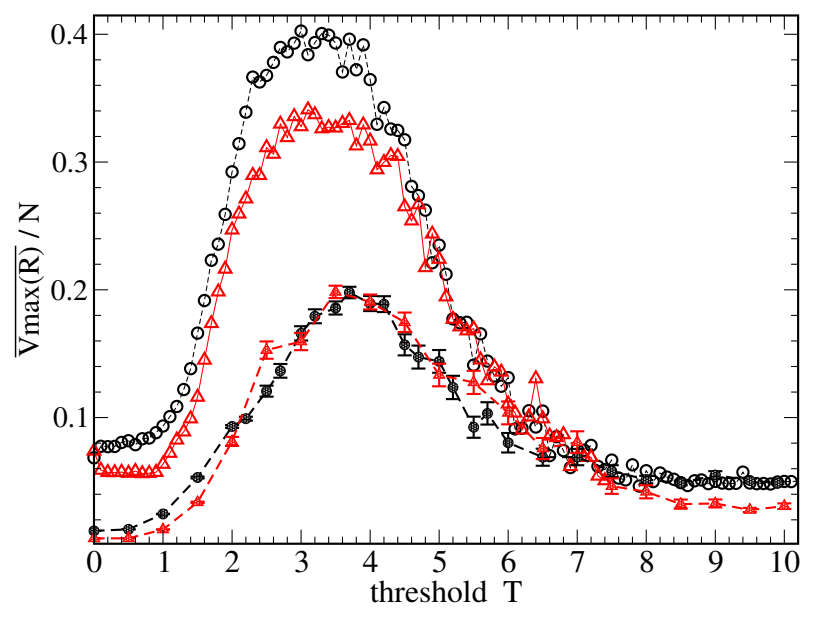

Fig. 1. The dominant commodity strength $V_{\max }(R)$ after the time range of observation of $R=1000$ turns, averaged over $n r=100$ realizations, versus the threshold $T$. The data for the present model are represented by filled symbols, whereas open symbols refer to results with the exchange protocol discussed previously in [9]. The two sets of data illustrate the quantitive significance of the adopted change. Results for the presently investigated model are plotted for various numbers of agents $N$ with the number of commodities $M=N$ : circles $-N=100$, triangles $-N=200$. Error bars correspond to a single standard deviation, estimated for the $n r=100$ realizations. Note that, for the purpose of this comparison, the dominant commodity strengths have been rescaled by the parameter $N$.

The figure presents a general outlook on the dominant commodity emergence from the "barter trade" phase to the phase of a "single universal commodity" in the sense given by Eq. (2). The strong ordered phase (the shape and position of the maximum) with clearly pronounced dominant commodity weakly depends on $N$. This weak dependence is due to a system finite-size effects and considered time of the commodity market simulations.

One can see that the dominant commodity strength $V_{\max }(t)$ as defined by Eq. (2) clearly indicates a regime of threshold values for approximately $2 \leq T \leq 6$. Particular modification in the exchange protocol decreases the maximum value of the commodity strength which could be reached with the threshold variation. However a general qualitative model behavior seems to be unchanged.

It means that the general agent-based model, considered here, is to some extent robust with respect to variations of its microscopic rules governing the agents' interactions. Obviously this is by no means a total surprise, as there is a great deal of known examples of complex physical systems, where for certain regimes of parameters, the particular nature of interactions is not important when one is interested in studying universal statistical properties (see e.g. [10]).

Now let us discuss the problem of the influence of finitesize model effects on the results obtained. Figure 1 suggests that the finite-size effects could be less pronounced

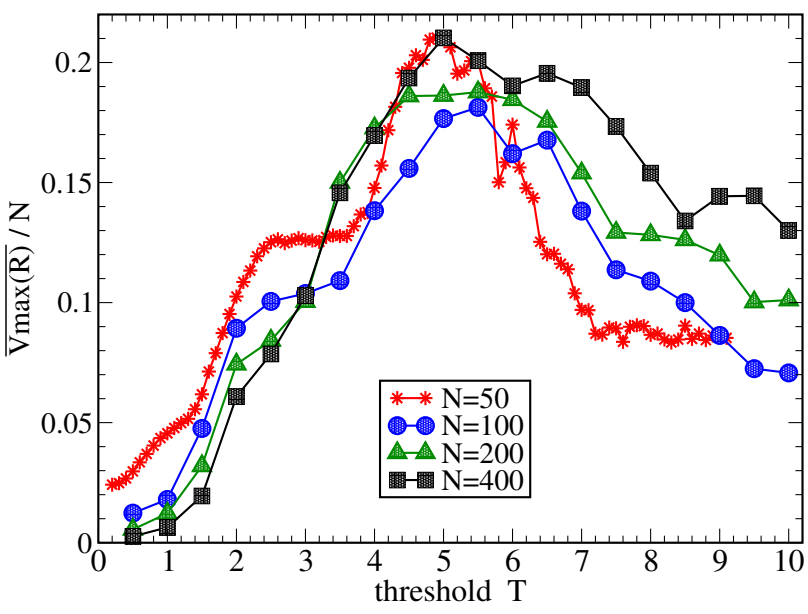

Fig. 2. The dominant commodity strength $V_{\max }(R)$ after the time range of observation of $t=R=10^{4}$ turns, averaged over $n r=100$ realizations, versus the threshold $T$. Data shown for various numbers of agents $N$ equal to the number of commodities $M(N=M)$ in the model: asterisks correspond to $N=50$, circles $-N=100$, triangles $-N=200$, and squares $N=400$. Note that, for the purpose of this comparison, the dominant commodity strengths have been rescaled by the parameter $N$.

in the model with changed interactions. The difference between maximum attainable values of the dominant commodity strength for the case of $N=M=100$ and $N=M=200$ seems to be less pronounced for the case of agents practising value-for-value exchange than for the case of just one-to-one exchange. Nevertheless, the variation with the model size is still visible if we compare results of our present model for a wider range of $N$ values - see Fig. 2, showing the averaged dominant commodity strengths in the case of 10 times longer evolution times.

This observation brings some fundamental questions about existence of the thermodynamic limit to a local or the global equilibrium state, and possibly the rate of convergence to such states, with the model size $N=M$ growing.

Figure 2 presents the averaged commodity strength after $R=10^{4}$ turns of the system evolution. The figure shows a comparison for different sizes of the model considered, ranging from $N=M=50$ up to $N=M=$ 400. The range of threshold values for which significant strength of the dominant commodity is allowed, is similar for all considered here model sizes at chosen observation time of $R=10^{4}$ turns. Again averaging of results at the end of the observation time is carried out over $n r=100$ realizations.

Although the qualitative agreement of these results is quite satisfactory, a question still remains open as to the convergence rate with growing values of $N=M$. Note that the agreement between the dominant commodity strengths corresponding to different $N=M$ values in the case of a longer time of observation is somewhat 
worse than for the case of a shorter observation time (see Fig. 1). This suggests presence of various regimes of behaviour over different time scales in our model commodity market evolution.

\subsection{The dynamics of commodity strengths}

In order to investigate further the dynamics of the system over different time scales, let us focus on the maximum shape variability with growing observation time of our model economy. We have already discussed in the previous subsection some indications, that this maximum is a signature of a sort of dynamically ordered phase where a single commodity value is well pronounced. Hence to support that tentative conclusion, let us consider three different observation times: $R=10^{3}, R=$ $10^{4}$, and $R=10^{5}$ turns for the smallest model size shown in Fig. 2. The results of the comparison are illustrated in Fig. 3.

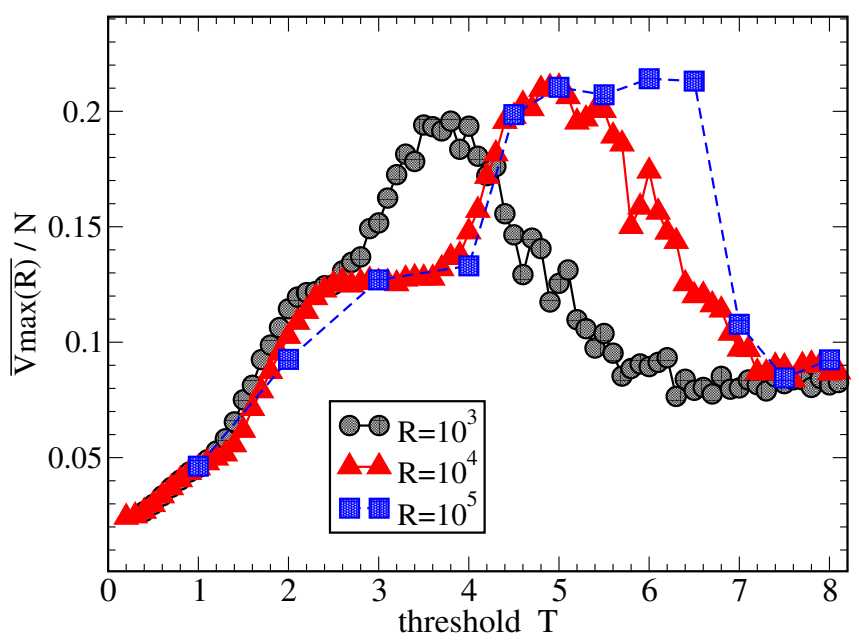

Fig. 3. The dominant commodity strength $V_{\max }(R)$ after time ranges of observation of $R=10^{3}$ turns (circles), $R=10^{4}$ (triangles), and $R=10^{5}$ (squares) averaged over $n r=100$ realizations, versus the threshold $T$. Data shown in the case when the number of agents is equal to the number of different types of commodities, $N=M=50$.

From inspection of Fig. 3 it follows that the maximum shape of the characteristics evolves in time, with the maximum value being approximately the same over five orders of magnitude of evolution times. Additionally, it appears that the sensitivity to time range considered is much greater for $T>3$ than it is the case for $T<3$. Indeed, such time variation of the dominant commodity strenghs is shown in Fig. 4 for a few interesting values of the threshold $T$. Data corresponding to a fixed value of $T$ show averaged $V_{\max }$ as a function of time. Again $n r=100$ realizations have been taken. Note that within observation time of $10^{5}$ turns, dominant commodity strengths possible to obtain for thresholds from $T=2.0$ up to $T=5.0$ already passed their "prosperity period" (the maximum build over time evolution), whereas model commodity market characterised by the threshold value of $T=8$ (orange line) is on the rising trend and needs still more time to build at least a local maximum of the dominant commodity strength. Such finite periods of prosperity which do not overlap in time for some ranges of threshold values cause variation of the maximum shape, seen in Fig. 3. This is yet another indication of a possible mechanism of the dominant commodity competition over time, whereby some hierarchy of commodity strengths is formed and remains for a period of time and then it reorders (e.g. another commodity takes a dominant role).

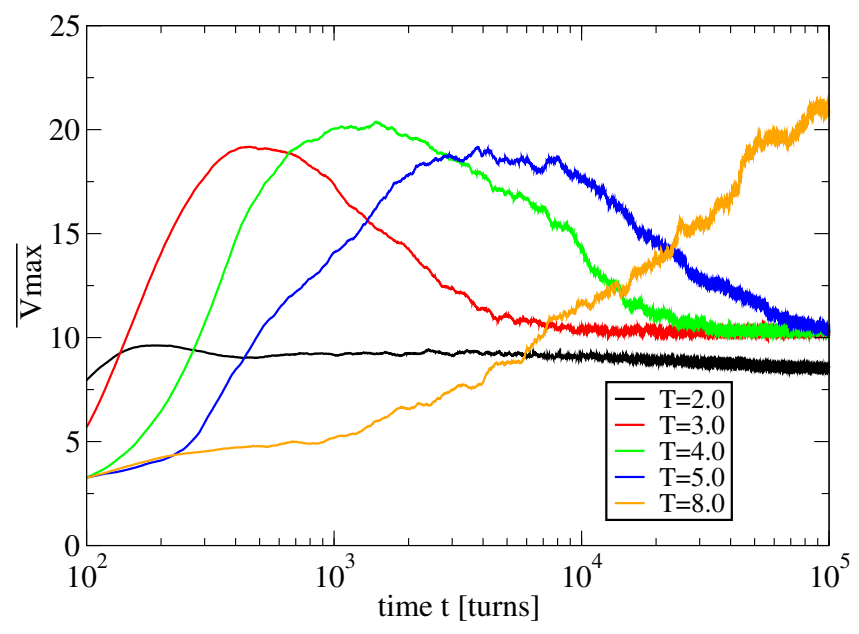

Fig. 4. The dominant commodity strength $V_{\max }$ versus time, averaged over $n r=100$ realizations. The agentbased computations are carried out for $N=M=100$ The data shown for a few values of the threshold $T$ : $T=2.0$ (black line), $T=3.0$ (red line), $T=4.0$ (green line), $T=5.0$ (blue line) and $T=8.0$ (orange line).

Data shown in Fig. 4 suggest that the higher value of the threshold, the more time needs the system to manifest ordered phase of a dominant commodity. That explains to some extent why it is easier to observe for a given evolution time a better convergence for smaller values of the threshold parameters (see Fig. 1 and Fig. 2). This model property could be in some analogy to the socalled critical slowing down problem due to growing correlations in the system, typical in some Monte Carlo simulations near the phase transition regime [11, 12]. Note also increasing role of fluctuations when time evolution exceeds range of about $10^{4}$ turns.

\subsection{On scaling properties in the agent-based model}

We have already argued that for various reasons convergence of the model by taking a limit of just a single parameter $N=M$ may be a too difficult question to answer due to the intrinsic dynamics of the dominant commodity, which depends also on the threshold value $T$. It is well known that the finite-size effects may have some significant influence on results of the simulations. Such various universal scalings are known analytically for some exactly-solvable models (e.g. for some variants of 
the Ising model) and could be investigated also numerically $[11,12]$. For example, a recent numerical study shows applications of such scalings and finite-size effects in the presence of an external Gaussian noise [13], which modifies the interactions in the system. It illustrates in that way the influence of the change of interactions on the critical parameters of the phase-transition in the Ising model on a square lattice.

Unravelling similar type of scalings in statistically derived observables of presently studied agent-based model would allow to check the model convergence in proper, rescaled variables. However, this daunting task requires extensive numerical simulations of the present model, or its redefinition so as to be able to tackle it with some analytical methods allowing to solve it at least approximately. Nevertheless, in order to check the feasibility of such approach one could consider some special cases and conjecture particular scalings.

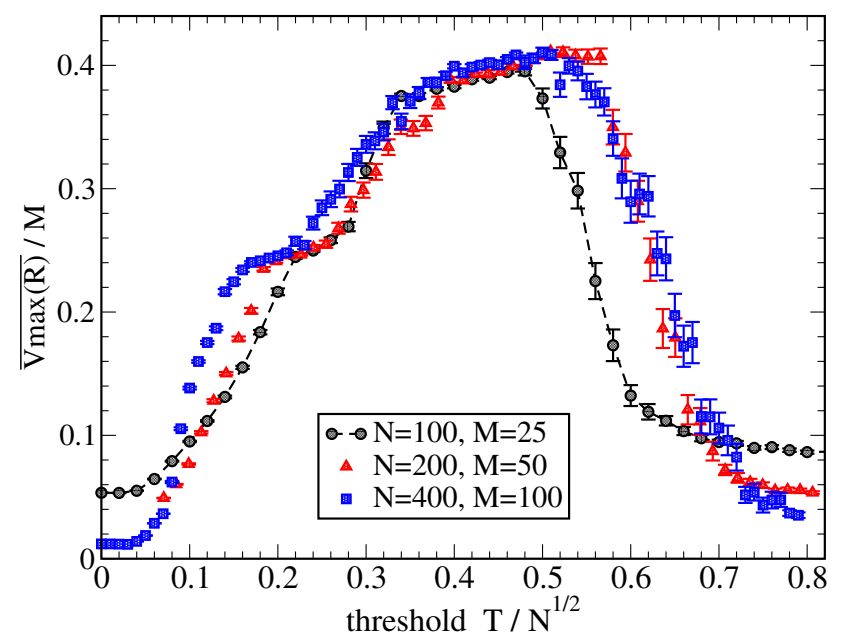

Fig. 5. The dominant commodity strength $V_{\max }$ versus threshold after $R=10^{4}$ turns, averaged over $n r=100$ realizations. The agent-based computations are performed for a fixed ratio $N: M=4: 1$ of the number of agents to the number of different types of commodities. Under conjectured scaling $\left(V_{\max }(R)\right.$ with $\left.M\right)$ and threshold $T$ (with $\sqrt{N}$ ) there is approximate collapse of data, obtained for different combinations of $N$ and $M$ values: $N=100, M=25$ - circles; $N=200, M=50$ — triangles and $N=400, M=100$ - squares. Error bars show numerically estimated dispersion of results, corresponding to a single standard deviation.

Figure 5 illustrates the case of unequal number of agents $N$ and types of commodities $M$. The figure allows to envisage to what extent such collapse of data may be achieved, provided a resonable scaling is conjectured. The considered case corresponds to a fixed ratio of $N: M=4: 1$. The figure shows results for different relevant cases, namely: $N=100, M=25$; $N=200, M=50$ and $N=400, M=100$. When rescaled in such a way that $\overline{V_{\max }} \rightarrow \overline{V_{\max }} / M$ and threshold $T \rightarrow T / \sqrt{N}$, the three different sets of data ap- proximately collapse to a single universal characteristics. Error bars denote the standard deviation of the mean values, averaged over $n r=100$ realizations. Note that rescaled data for $N=200$ and $N=400$ collapse reasonably well between 0.3 and 0.7 in rescaled threshold $T$ values. Figure 5 also suggests that the rescaled data collapse the better the higher number of agents is taken.

\subsection{Towards emergence of commodity exchange rates}

Finally, let us consider a case where $N \gg M$ which is a typical situation for real commodity markets. The example shown in Fig. 6 corresponds to the case of $N=10^{4}$ agents and $M=100$ commodities. The figure shows a temporal variation of a single realization for two arbitrary chosen commodity types. Their strengths $V_{1}, V_{2}$, and $V_{3}$ are taken relative to another commodity strength, that is they are expressed in terms of strength units of another type of the commodity (not shown). This mimics to some extent mutual exchange rates in the toy commodity market (albeit with more realistic size than it has been investigated previously). Note the competition of emerged exchange rates $V_{2}$ and $V_{3}$, shown over time of more than 5000 turns.

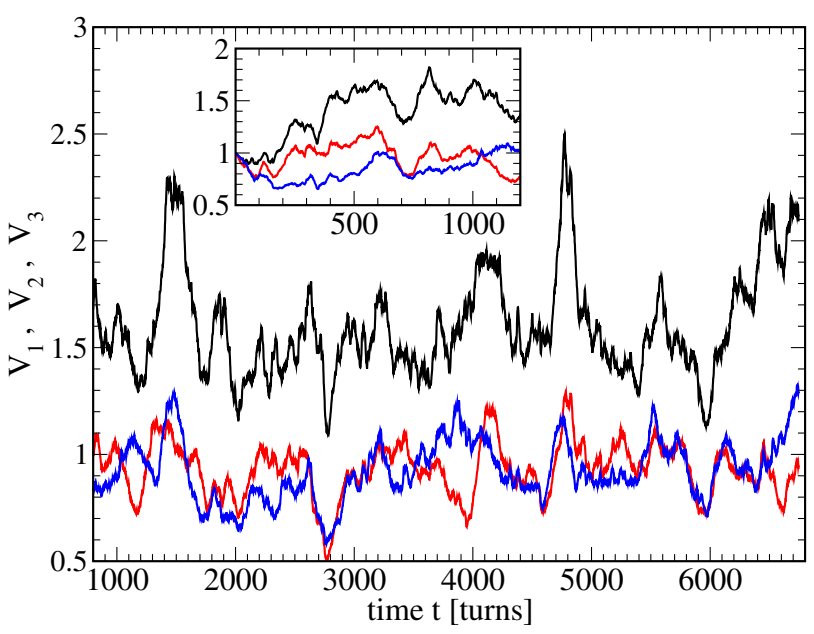

Fig. 6. Commodity exchange rates for $N=10^{4}$ and $M=100$ (a regime of parameters where $N \gg M)$. The commodity strengths $V_{1}$ (black curve) and $V_{2}$ (red curve) and $V_{3}$ (blue curve) are expressed in terms of $V_{5}$ (not shown) for a single realization of the economy scenario over time range of $R=6700$ turns. Note a competition between such defined commodity exchange rates, showing itself as a reversal of relative exchange rates (the second or the third commodity becomes more valuable in terms of the fifth, base commodity during the evolution time).

The inset of Fig. 6 shows initial system evolution over first 1000 turns, to allow for a better inspection of short time scale behavior and the way such competition of exchange rate values sets in. There are periods when each of these two commodities takes in turn a higher rate expressed in some other, common (base) commodity units, possibly after periods of being equivalent to each other. 


\section{Summary and conclusions}

In this paper we have considered a modification of microscopic rules, governing the exchange between interacting economic agents on a toy commodity market. On one hand, allowing value-for-value exchange instead of one-to-one swap mechanism brings our toy model closer towards more realistic agent-based economic system. Therefore it is of practical importance for real commodity market modelling. It has been argued here that in principle such a modification admits a possibility of exchange rate emergence in the system, allowing to express more directly the link between exchange rates and the competition of commodities for the dominant status. On the other hand, the experience gained with the modified model gives some feeling about sensitivity of that particular model on details of interactions near the critical regime of behavior. This might be of some fundamental interest related to the universality classes of agent-based models, which could be expressed in terms of scalings with respect to the finite size of the model.

\section{Acknowledgments}

One of us (R.G.) would like to acknowledge the use of the services and computer resources provided by the Academic Computer Center CYFRONET AGH in Kraków (Akademickie Cen- trum Komputerowe CYFRONET AGH, Grant No. MNiSW/IBM_BC_HS21/PK/033/2014).

\section{References}

[1] D. Sornette, Rep. Prog. Phys. 77, 062001 (2014).

[2] T. Lux, M. Marchesi, Nature 397, 498 (1999).

[3] J. Voit, The Statistical Mechanics of Financial Markets, 3rd ed., Springer, Berlin 2005.

[4] V.M. Yakovenko, J.B. Rosser, Jr., Rev. Mod. Phys. 81, 1703 (2009).

[5] A. Yasutomi, Physica D 82, 180 (1995).

[6] A. Yasutomi, Chaos 13, 1148 (2003).

[7] A.Z. Górski, S. Drożdż, P. Oświęcimka, Acta Phys. Pol. A 117, 676 (2010).

[8] P. Oświęcimka, S. Drożdż, R. Gębarowski, A.Z. Górski, J. Kwapień, Acta Phys. Pol. B 46, 1579 (2015).

[9] R. Gębarowski, S. Drożdż, A.Z. Górski, P. Oświęcimka, Acta Phys. Pol. A 127, A-51 (2015).

[10] J. Kwapień, S. Drożdż, Phys. Rep. 515, 115 (2012).

[11] K. Binder, Z. Phys. B 43, 119 (1981).

[12] K. Binder, Rep. Prog. Phys. 60, 487 (1997).

[13] R. Gębarowski, Techn. Trans. Fundam. Sci. 3-NP, 51 (2014). 